\title{
BMJ Open Prevalence and determinants of undetected dementia in the community: a systematic literature review and a meta-analysis
}

\author{
Linda Lang,,$^{1,2}$ Angela Clifford, ${ }^{1}$ Li Wei, ${ }^{3}$ Dongmei Zhang, ${ }^{4}$ Daryl Leung, ${ }^{5}$ \\ Glenda Augustine, ${ }^{6}$ Isaac M Danat, ${ }^{1}$ Weiju Zhou, ${ }^{1}$ John R Copeland, ${ }^{7}$ \\ Kaarin J Anstey, ${ }^{8}$ Ruoling Chen ${ }^{1,2}$
}

To cite: Lang L, Clifford A, Wei $L$, et al. Prevalence and determinants of undetected dementia in the community: a systematic literature review and a meta-analysis. BMJ Open 2017;7:e011146. doi:10.1136/bmjopen-2016011146

- Prepublication history and additional material is available. To view please visit the journal (http://dx.doi.org/ 10.1136/bmjopen-2016011146).

$A C$ is co-first author.

Received 18 January 2016 Revised 7 December 2016 Accepted 11 January 2017

CrossMark

For numbered affiliations see end of article.

Correspondence to Dr Ruoling Chen, r.chen@wlv.ac.uk; Dr Li Wei, I.wei@ucl.ac.uk and Dr Dongmei Zhang (for Chinese), zdmd@sina.com

\section{ABSTRACT}

Objectives: Detection of dementia is essential for improving the lives of patients but the extent of underdetection worldwide and its causes are not known. This study aimed to quantify the prevalence of undetected dementia and to examine its correlates.

Methods/setting/participants: A systematic search was conducted until October 2016 for studies reporting the proportion of undetected dementia and/or its determinants in either the community or in residential care settings worldwide. Random-effects models calculated the pooled rate of undetected dementia and subgroup analyses were conducted to identify determinants of the variation.

Primary and secondary outcome measures: The outcome measures of interest were the prevalence and determinants of undetected dementia.

Results: 23 studies were eligible for inclusion in this review. The pooled rate of undetected dementia was $61.7 \%(95 \% \mathrm{Cl} 55.0 \%$ to $68.0 \%)$. The rate of underdetection was higher in China and India (vs Europe and North America), in the community setting (vs residential/nursing care), age of $<70$ years, male gender and diagnosis by general practitioner. However, it was lower in the studies using Mini-Mental State Examination (MMSE) diagnosis criteria.

Conclusions: The prevalence of undetected dementia is high globally. Wide variations in detecting dementia need to be urgently examined, particularly in populations with low socioeconomic status. Efforts are required to reduce diagnostic inequality and to improve early diagnosis in the community.

\section{INTRODUCTION}

Detecting people living with dementia is crucial for necessary care and treatment. Early diagnosis allows for advanced-care planning and improves prognosis. ${ }^{12}$ However, the level of dementia detection in the population may be low, especially as many older adults experience memory decline and changes in

\section{Strengths and limitations of this study}

- To the best of our knowledge, this is the first systematic synthesis of data available globally to estimate the rate of underdetection of dementia in people.

- Owing to the lack of data we could not include any study from low-income countries, such as those in Africa or South America, to investigate the prevalence and determinants of undetected dementia.

- We did not include additional factors which may affect the detection of dementia in the metaanalysis because too few studies had sufficient or comparable data for each analysis, though we did include them for general discussion.

brain activity as part of the normal ageing process. ${ }^{3}$ Some studies have shown that more than half of people living with dementia in the community are not detected ${ }^{4} 5$ while others reported that the proportion of undetected dementia could exceed $90 \% .^{6}$ In spite of the uncertainties around the estimated proportion of undetected dementia and its determinants, no systematic literature review has been carried out on the underdetection of dementia in the worldwide population. We know little about the extent of underdetection globally as well as the causes of this problem. Understanding the occurrence of and factors influencing the proportion of undetected dementia is important for improving detection rates, health policy and planning, and the well-being of patients and their families through access to appropriate support services. For the foreseeable future, policy directives in dementia care are likely to be aimed at early diagnosis of dementia. We conducted a meta-analysis to assess the proportion of undetected dementia in people 
with dementia across the world and identify factors influencing the detection of dementia.

\section{METHODS}

\section{Literature search}

We searched the databases MEDLINE, Web of Knowledge, Science Direct and Google Scholar (date range unlimited) to identify studies eligible for this review. The primary search terms included combinations of the following keywords: 'undetected, detection, undiagnosed, diagnosis, dementia and Alzheimer's disease'. The full texts of articles that reported the rate of detection or underdetection of dementia and/or determinants of detection were retrieved for further assessment. We also manually searched the bibliographies of selected papers for additional articles. The literature search was completed in October 2016 (by ID and WZ, following the initial search completion by AC and LL in July 2013).

To be eligible for this systematic review, each study must have identified a sample of people with dementia using an independent assessment for dementia delivered by the researchers (and independent from general practitioners (GPs)). Studies must then have compared this assessment of dementia with the medical records held by participants' GP or other primary care facility to determine if the dementia had been detected by the healthcare system. Those studies, which used a knowledgeable informant, family report of dementia or a nurse confirming which patients have dementia, were not included in this review, for example, the study of Savva and Arthur. ${ }^{7}$ Other standards of comparison (eg, caregiver recognition of memory problems, different screening tools) were also excluded from this meta-analysis and only formal medical records were considered for comparison with the independent assessment (some such studies ${ }^{18}$ are included in the general discussion). Studies were excluded if the sample was selected or identified for having a specific health characteristic that was not dementia (eg, delirium ${ }^{9}$ or based on admission to psychiatric hospital ${ }^{10}$ ) as this may have biased the pooled estimate. One further study was excluded as the whole sample had been referred to a memory clinic for memory problems, implying some awareness of memory problems in all participants. ${ }^{11}$ We removed any duplicated publication of a study ${ }^{12}$ and used the first publication. ${ }^{13}$ Review articles were excluded, as were articles that did not provide sufficient information for the purposes of this review such as letters, citations and conference information. Samples from both the community and/or residential care settings were included. Online supplementary figure S1 shows the study selection process that identified 23 eligible studies.

\section{Literature review}

We followed MOOSE guidelines for systematic literature reviews ( $\mathrm{AC}$ and $\mathrm{LL}$ did it for the identified studies published before July 2013, and ID and WZ for others). Information was extracted from eligible articles based on predefined criteria. We extracted information on the year of publication, the study design, how participants were recruited (or the name of the study where available), the country in which the study was conducted, the number of participants, the mean age of the participants at the time of cognitive testing, participant gender, the method of diagnosis, the covariates used in the analyses, the percentage of undetected dementia in the sample and the determinants examined with their findings. Eligible articles were reviewed for quality using a modified version of the Newcastle-Ottawa criteria, ${ }^{14}$ suggesting that the quality of these articles was good, without any study being excluded for meta-analysis. To explore the determinants we used meta-analysis as described below. We did not include determinants in the meta-analysis if too few studies had sufficient or comparable data for each variable. However, we carried out a qualitative review of these additional variables in the Discussion section of this paper.

\section{Meta-analysis}

To be eligible for the meta-analysis, studies must have reported the rate of undetected dementia within a sample. Using methods employed in previous studies, ${ }^{15}$ we ran a meta-analysis to estimate the proportion of undetected dementia. The pooled estimate was calculated using weights based on inverted variances of estimates from each study sample. The fixed-effects model or random-effects model was employed according to a statistical test of the homogeneity assumption- $Q$ value. If heterogeneity of within-study and between-study variation in those selected studies was significant $(p<0.05)$, a random-effects model was used; otherwise, a fixed-effects model was used. We performed additional subgroup analyses to examine variation in the prevalence of undetected dementia by country, study setting and study characteristics such as the minimum age and sex ratio of the studied population, different ways of detecting and diagnosing dementia, and earlier versus later studies. All analyses in this study were performed in the R statistical package (V.3.0.1).

\section{RESULTS}

The characteristics of these 23 studies identified ${ }^{6} 1316-36$ are shown in online supplementary tables S1 and S2 according to the study settings. They were published between 1988 and 2015; five studies were published before 2000, six published in 2000-2004, seven in 20052009 and five in 2010-2015. They were mostly conducted in Western Europe $(n=11)$ or North America $(\mathrm{n}=10)$, and only two studies were conducted in middle-income countries in Asia (Thailand, China). ${ }^{6} 28$ No study was found from low-income countries (classified according to the World Bank ${ }^{37}$ ). Fifteen studies recruited community-dwelling older people, while five 
focused on residential settings ${ }^{31} 32{ }^{34-36}$ and a further three studies recruited participants from both community and residential populations. ${ }^{13} 23{ }^{24}$ Studied populations varied from $13^{17}$ to $39692 .{ }^{36}$ Mean age in studies (where given) ranged from $67^{28}$ to 84 years ${ }^{31}$ and all samples (where information given) included both men and women. The majority of articles $(n=17)$ used medical records to examine existing diagnoses of dementia; however, there was a wide range of different methods employed to screen for dementia within the sample for the purposes of the research. The characteristics of each of the 23 studies are shown in online supplementary tables S1 and S2 according to the study settings. The narrative review of underdetection and its possible determinants in community-based studies $(n=15)$, in residential-based studies $(n=5)$, and in mixed community and residential-based studies $(n=3)$ have been included in online supplementary appendix 1 . They suggested that there was a big variation in the rate of underdetection among 23 studies (from $31 \%$ to $96 \%$ ), which may be related to the studies' locations (countries and settings), year of publication, age and sex of the populations, and methods of detecting and diagnosing dementia.

Figure 1 shows the variation in the rate of undetected dementia in the 23 studies and the pooled rate of underdetection. There were a total of 30332 undetected dementia cases in 43446 people living with dementia. The random-effects model analysis gave a prevalence rate of undetected dementia as $61.7 \%$ (95\% CI $55.0 \%$ to $68.0 \%)$. Recognising the high influence of Bartfay and colleagues' study to the meta-analysis model, excluding this study from the analysis gave a pooled proportion of $61.3 \%$ (53.8\% to $68.3 \%$; random-effects model due to its heterogeneity $\mathrm{p}<0.001$ ), which is not substantially different from the pooled proportion for all studies.

Tables 1 and 2 show the rate of underdetection of dementia in subgroup data analysis. The rate of undetected dementia was significantly increased in lower income countries $(93.2 \%$ in Asia, $62.9 \%$ in North America, $53.7 \%$ in Europe), and in the communitybased settings $(63.6 \%$ vs $50.9 \%$ in the nursing residential homes; table 1). The rate of underdetection was higher among younger ages, and among populations with higher proportions of men. Detection of dementia by GPs was associated with increased underdetection. Using Diagnostic and Statistical Manual of Mental Disorders Third/Fourth Edition (DSM-III/IV) criteria to identify dementia was associated with a higher rate of underdetection than screening with the Mini-Mental State Examination (MMSE). When the studies were split into two groups based on publication date, there was a nonsignificant increase in the underdetection rate in studies published since 2005 compared with those published earlier (table 2).

\section{DISCUSSION}

Our systematic literature review identified that studies reporting underdetection of dementia in the community were mainly from high-income countries and no study was undertaken in low-income countries. The

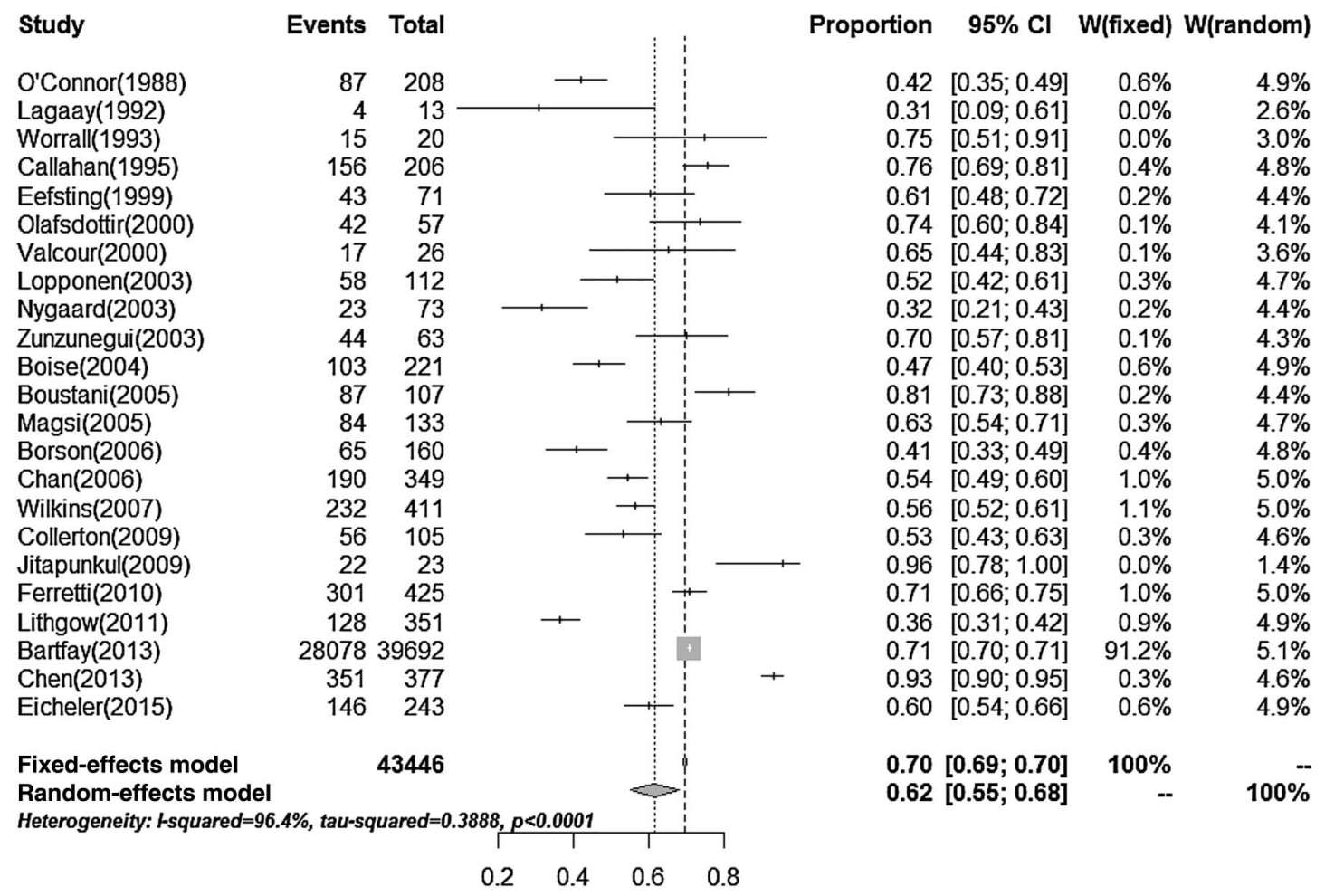

Figure 1 Forest plot for these 23 studies. 
Table 1 Meta-rate of undetected dementia by location of study

\begin{tabular}{|c|c|c|c|c|}
\hline Location of study & $\begin{array}{l}\text { Number } \\
\text { of studies }\end{array}$ & $\begin{array}{l}\text { Number } \\
\text { of patients }\end{array}$ & $\begin{array}{l}\text { Number } \\
\text { of cases }\end{array}$ & $\begin{array}{l}\text { Meta-prevalence } \\
(\%(95 \% \mathrm{Cl}))\end{array}$ \\
\hline \multicolumn{5}{|l|}{ Country* } \\
\hline UK & 3 & 664 & 271 & 43.1 (34.6 to 52.0$)$ \\
\hline Those in Europe except UK & 8 & 1057 & 661 & $58.2(48.3$ to 67.5$)$ \\
\hline USA & 8 & 1613 & 934 & 60.7 (51.7 to 69.0$)$ \\
\hline Canada & 2 & 39712 & 28093 & 70.7 (70.3 to 71.2$)$ \\
\hline Those in Asia & 2 & 400 & 373 & 93.2 (90.3 to 95.3$)$ \\
\hline \multicolumn{5}{|l|}{ Setting } \\
\hline Community-based only & 15 & 2816 & 1776 & 63.7 (54.8 to 71.8$)$ \\
\hline Residential or nursing care only & 5 & 40249 & 28313 & 50.9 (29.5 to 71.9$)$ \\
\hline Population study (community and/or residential) & 3 & 381 & 243 & 65.8 (49.7 to 79.0$)$ \\
\hline
\end{tabular}

Table 2 Undetected dementia in relation to other factors

\begin{tabular}{|c|c|c|c|c|}
\hline Variable & Number of studies & Number of patients & Number of cases & Meta-prevalence $(\%(95 \% \mathrm{Cl}))$ \\
\hline \multicolumn{5}{|c|}{ Minimum age of studied patients in the study } \\
\hline$<70$ & 8 & 1333 & 966 & $74.6(60.4$ to 85.0$)$ \\
\hline 70-79 & 7 & 1237 & 738 & $61.9(51.0$ to 71.7$)$ \\
\hline$\geq 80$ & 2 & 118 & 60 & 45.8 (26.3 to 66.7$)$ \\
\hline Unknown & 6 & 40758 & 28568 & 49.8 (34.4 to 65.3 ) \\
\hline \multicolumn{5}{|c|}{ Per cent of females in the study } \\
\hline$\geq 65$ & 9 & 41544 & 29196 & 62.1 (54.3 to 69.3$)$ \\
\hline$<65$ & 5 & 810 & 643 & 78.4 (58.7 to 90.2$)$ \\
\hline Unknown & 9 & 1092 & 493 & 49.4 (40.0 to 58.8$)$ \\
\hline \multicolumn{5}{|c|}{ Methods of dementia diagnosis } \\
\hline GP & 4 & 899 & 627 & 68.4 (40.8 to 87.2$)$ \\
\hline Medical record & 16 & 2371 & 1415 & 60.6 (53.2 to 67.4$)$ \\
\hline Facility record & 2 & 39825 & 28162 & 68.1 (60.7 to 74.7$)$ \\
\hline Care plan & 1 & 351 & 128 & 36.5 (31.4 to 41.7$)$ \\
\hline \multicolumn{5}{|c|}{ Identification of dementia } \\
\hline MMSE & 6 & 1270 & 719 & 54.3 (40.7 to 67.3$)$ \\
\hline DSM-III/IV & 5 & 326 & 209 & 67.0 (54.6 to 77.5$)$ \\
\hline ICD-10 & 2 & 180 & 110 & 58.6 (13.5 to 92.8$)$ \\
\hline CASI & 2 & 186 & 82 & 51.4 (28.3 to 73.9$)$ \\
\hline CERAD & 2 & 632 & 335 & 51.8 (42.2 to 61.3$)$ \\
\hline Others ${ }^{*}$ & 6 & 40852 & 28877 & 71.2 (58.2 to 81.4 ) \\
\hline \multicolumn{5}{|c|}{ Year of study publication } \\
\hline$<2005$ & 11 & 1070 & 592 & 57.2 (47.1 to 66.8$)$ \\
\hline$\geq 2005$ & 12 & 42358 & 29740 & 65.3 (56.5 to 73.2$)$ \\
\hline
\end{tabular}

*Others including CAMDEX, CMSQ, SPMSQ, neuropsychological battery, 10/66 and CPS.

CAMDEX, Cambridge Mental Disorders of the Elderly Examination; CASI, Cognitive Abilities Screening Instrument; CERAD, Consortium to Establish a Registry for Alzheimer's Disease; CMSQ, Canadian Mental Status Questionnaire; CPS, Cognitive Performance Scale; DSM-III/IV, Diagnostic and Statistical Manual of Mental Disorders Third/Fourth Edition; GP, general practitioner; ICD-10, International Classification of Diseases 10th Edition; MMSE, Mini-Mental State Examination; SPMSQ, Short Portable Mental Status Questionnaire.

meta-analysis showed that the proportion of undetected dementia is high; more than $60 \%$ of people with dementia are not detected in the community and/or residential/nursing care. The studies included in this review also showed wide variability in estimates, from one-third to around $95 \%$ of studied cases, and some determinants were identified. Although the rate was high in the community compared with residential settings, the rate of undetected dementia in residential care was still high at around $50 \%$. The rate of underdetection was inversely related to income of countries, with middle-income countries showing particularly high rates of underdetection (above 90\%) in comparison to around $60 \%$ in highincome countries, although this may be also due to the countries' cultural attitude to dementia or methodological features of the studies included from these regions. While individual studies showed contradictory findings, ${ }^{13} 202124273436$ our meta-analysis suggested that risk of undetected dementia among older adults is greater for men and for people with dementia at earlier 
ages. The rate of underdetection of dementia was also increased in the diagnosis by GP, but reduced using the MMSE criteria to identify dementia.

Our study has limitations. First, due to the lack of data we could not include any study from low-income countries, such as those in Africa or South America where some population-based research was carried out to investigate the prevalence and incidence of dementia. ${ }^{38}$ Thus, the findings cannot be applied to low-income countries. However, based on the meta-analysis findings from middle-income and high-income countries we consider that the underdetection of dementia in low-income countries could be higher. Thus, the current metaprevalence of underdetection of dementia in the community may be more conservative. Second, due to our selection criteria of the studies for review, we did not include the articles (which reported the underdetection of dementia but they depended on dementia recognition by family members rather than healthcare provi$\operatorname{ders}^{7}{ }^{8}$ ), and online reports, for example, the UK 'Putting Dementia on the Map' which states that the national average rate of dementia diagnosis is $48 \% .^{39}$ This would lead to a smaller number of studies to be included than expected, and wider confidence limits of the estimated underdetection. However, their findings are similar to those in our meta-analysis. Third, the variation in the methods of diagnosing dementia among these 23 studies made it less efficient to compare the prevalence of underdetection of dementia among different populations. Our findings of differences in the rate of undetected dementia in the community need to be further studied in a large-scale global survey, using the same method of diagnosing dementia.

\section{Factors associated with the underdetection of dementia in the community \\ Socioeconomic status and sociodemography within individual studies}

Although countries' data analysis showed a socioeconomic gradient with underdetection of dementia, few studies examined this within the study. In China, increased risk of having undetected dementia was strongly associated with low socioeconomic levels, in particular low levels of education, occupational class and income and living in a rural area. ${ }^{6}$ In North America, however, the four studies ${ }^{19} 212527$ did not find a significant association between socioeconomic status and detection of dementia. With such a finding, we consider that differences in detection of dementia between countries with different incomes could be at least partly explained by the national health policy, health service system, culture and educational level, apart from income. However, there is at present too little evidence from countries with lower incomes on which to base firm conclusions.

A few studies ${ }^{13} \quad 24 \quad 27 \quad 34$ examined the association between underdetection of dementia and age in the community, suggesting that the underdetection of dementia increased with age. This is in contrast to the meta-analysis results. The association of the dementia detection rate with age in older people requires further investigation in a large-scale cohort study. Our meta-analysis finding of the association between male gender and undetected dementia is different from some of the previous studies. ${ }^{20} 27$ The underdetection of dementia in men may be due to lower level of seeking clinical consultation and also there is a tendency to hide the person out of a sense of shame. Eichler et $a l^{29}$ further reported that in people with dementia who did not get a formal diagnosis of dementia at baseline, the impact of a positive screening outcome on improving the diagnosis of dementia was less in men than women. The ethnicity of patients did not appear to affect the rate of dementia detection. ${ }^{19} 2127$ While Boise et al ${ }^{23}$ included mostly Caucasian participants with underrepresentation of ethnic minority groups, Borson et $a l^{25}$ over-represented ethnic minorities, yet both studies found similar rates of undetected dementia ( $46 \%$ vs $41 \%)$. However, Borson $e t a l^{25}$ observed a high rate of underdetection in non-English speakers $(70 \%)$ compared with local language speakers $(55 \%)$ in the USA, suggesting barriers to diagnosis for some minority groups. Previous studies ${ }^{40} 41$ have shown that stigma attached to mental illness, and a lack of knowledge about mental illness and services may lead to decreased use of services by older people with dementia from minority ethnic groups and this should be addressed.

\section{Social support and care}

According to a survey of GPs in Sweden ${ }^{17}$ the problem most likely to trigger a dementia investigation is a relative's symptom about a patient's memory loss. However, our review found inconsistent evidence of an association between social support and detection of dementia in high-income countries, though few studies looked at this in detail. ${ }^{13} 27 \quad 34 \quad 36$ Ross $e t a l^{8}$ found that $21 \%$ of 191 community-dwelling elderly Japanese-American men with dementia had a family informant who failed to recognise a memory problem, suggesting that family members may not always be reliable informants (this study was not included in the meta-analysis as it depended on recognition by family members rather than healthcare providers). O'Connor $e t a l^{16}$ showed a relationship between caregiver strain and detection of dementia, indicating that caregivers may play a vital role in ensuring a timely diagnosis is made. High levels of social support and lack of caregiver awareness may thus in fact both aid and hinder detection in different communities, and identification of dementia may be enhanced through targeted and culturally sensitive screening.

Previous studies showed that there is a higher prevalence of dementia in older people living in institutions than at home. ${ }^{13}$ Yet around half of elderly with dementia living in care homes do not have a clear diagnosis written into their care plans. This may be partly due to poor 
levels of diagnosis in primary care prior to entering the nursing home; however, these findings also highlight low rates of recognition among care home staff. This lack of recognition means people may not receive the specialist care that they need, and may increase challenging behaviours due to having unmet needs. Nursing home staff should be fully equipped with knowledge and resources to detect dementia and support residents properly. One study included in the meta-analysis ${ }^{32}$ suggested that residents in settings where they are considered more independent, such as assisted living facilities, may be at higher risk of having undetected dementia. Thus, these older people should also be monitored for changes in cognitive function to ensure their ongoing needs are met.

Primary care and acute services must be encouraged to diagnose dementia when present even in the oldest old to prepare secondary care services and nursing homes to care for the patient's needs. Increased frequencies of visits to the GP were associated with detection in some studies based on forced choice of GPs, ${ }^{20}$ but this was not the case in other studies. ${ }^{13} 1922 \quad 25$ Further investigation is therefore needed to determine whether GPs may benefit from further resources or support in order to recognise dementia symptoms accurately and quickly and any such needs should be addressed as soon as possible, though the needs of individuals may differ between practices and cultures.

\section{GP detecting dementia and criteria of dementia}

Our meta-analysis suggested that there were increased risks of undetected dementia by GPs. van den Dungen et $a t^{42}$ suggest that sensitivity of GP diagnosis of dementia is low. It is unclear whether low detection rates are due to a lack of GP knowledge of dementia symptoms, insufficient screening practices or a purposeful decision not to diagnose. We consider that some GPs and health providers continue to see memory difficulties as an acceptable part of the normal ageing process rather than as a disability that requires specialist care and support.

The meta-analysis results suggested that studies which used DSM-III/IV criteria to identify dementia found a higher rate of the underdetection than those using the MMSE, likely due to the low scores on the MMSE having several possible explanations such as poor language, poor schooling and learning disabilities. GPs detecting dementia and criteria for dementia diagnosis included depression, which affected the MMSE score. There are also other possibilities that the studies that defined (identified) dementia for the study using MMSE likely would have included many participants who in fact did not have dementia. This raises a methodological question for future research to allow for improved comparability across studies.

Clinical detection of dementia poses many practical problems at all levels from patient and relative awareness through to reluctance of clinical staff to engage. Historically there has been a lack of training and education of professionals to help them make the diagnosis of dementia. Traditional memory clinics run by psychiatrists in old age are often seen as the gold standard but have lacked capacity to cope with very large numbers. In addition, reluctance of GPs and hospital doctors to refer to memory clinics has resulted in underdiagnosis. Practically, a sound clinical history from a reliable source together with the exclusion of conditions mimicking dementia, for example, depression, delirium, stroke, medications and other brain diseases, should establish the diagnosis. The use of a reliable history from patients and/or collateral source, a recognised cognitive screening instrument, brain imaging, and routine blood tests would certainly improve the ability to detect dementia in older people.

\section{Other influencing factors from the qualitative review}

Among the 23 identified studies, some but not all, included additional factors which may affect the detection of dementia but which would require further investigation below (also see online supplementary table S3).

\section{Nature of cognitive impairment and comorbidity}

Although there is uncertainty around the differences in the detection rate between Alzheimer's disease and vascular dementia, ${ }^{13}{ }^{25}$ detection of dementia consistently improved with increased severity across studies. ${ }^{16} 18$ 20-23 25273033 Since mild-to-moderate symptoms are more likely to be undetected especially where contact with the GP is low, ${ }^{20}$ focus should be placed on identifying these cases through community awareness programmes. Interestingly, only one study investigated family history of dementia as a determinant of detection, finding no significant association. ${ }^{13}$ One study ${ }^{36}$ suggested an association between undetected dementia and other conditions, including schizophrenia and hearing problems. However, there is uncertainty around the link between undetected dementia and comorbid depression; two studies found a possible link ${ }^{13}{ }^{36}$ while the other two found no difference in the Geriatric Depression Score (GDS) score between detected and undetected cases. ${ }^{21} 34$ Depression is treatable but can mimic dementia which is comparatively untreatable. It is thus important that depression is not confused with dementia. However, the severely demented or severely depressed may not be able to complete the GDS satisfactorily.

The rate of undetected dementia was inversely associated with functional impairment, measured using activities of daily living scales. ${ }^{13} 2127{ }^{34}$ In a study not eligible for this meta-analysis (as medical records were not searched but rather patients were asked if they had seen a doctor for memory problems), Sternberg et al observed that those with no or few physical or functional difficulties were less likely to consult a GP about a memory problem. Such difficulties may act as warning signs of an underlying condition, prompting further investigation and subsequent diagnosis of cognitive impairment where present. The use of functional impairments may be useful to help improve diagnosis 
rates especially in those with mild cognitive symptoms, and a combined screening tool could be considered for those not directly seeking medical advice for these difficulties, perhaps for use by community nurses, pharmacists and other non-physician primary care providers.

Our study has suggested that current prevalence estimates tend to underestimate the true prevalence of dementia in the world. Particularly, it would happen in some countries experiencing epidemiological transition, such as China. ${ }^{43}$ As a consequence, planning for health and care services is likely to be inadequate and this has strong implications for public policy. The findings also suggest that there is a very large amount of unmet need in the community among older adults with dementia and their families. To the best of our knowledge, this is the first synthesis of data available globally to estimate the rate of underdetection in people with dementia, and the determinants of underdetection. Identification of inequality in detection of dementia will help improve the early detection of dementia by improving understanding of factors that hinder/promote diagnosis and by identifying areas needing resources and training. For example, this knowledge could lead to targeted awareness campaigns for younger age groups and targeted screening for men or non-native speakers, which could help to improve diagnosis rates in these groups. Primary and acute care providers should receive training and support in identifying and diagnosing early symptoms of dementia, before the onset of severe daily impairments, and awareness of the benefits of early diagnosis should be increased. Detection can also be improved through closer liaison with specialists, better use of corroborative history from family members and including dementia in comprehensive assessments of older adults by GPs. While mild memory impairments such as 'difficulty recalling names' may be part of normal ageing, more serious forgetfulness causing social embarrassment, especially if the person appears unaware of any difficulty, should arouse suspicion and the need for diagnosis and early intervention. Efforts to allow the organisation of adequate healthcare provisions for older people to improve the detection of dementia should be made a priority globally.

\section{CONCLUSION}

In this systematic literature review and meta-analysis, we have identified that the proportion of underdetection of dementia in the world is high and varies among countries. The underdetection of dementia may be associated with low income, and with younger age and male gender. Its inverse association with functional impairment may reflect the delay in detecting dementia in older populations. While there are currently no recommendations for the screening of older adults for dementia in many countries, our findings suggest that certain high-risk groups, for example, those of low socioeconomic status, non-local language speakers and those who live alone should be offered screening to enable a fast diagnosis and for treatment to be offered as early as possible.

\section{Author affiliations}

${ }^{1}$ Faculty of Education, Health and Wellbeing University of Wolverhampton, Wolverhampton, UK

${ }^{2}$ Post Graduate Academic Institute of Medicine, University of Wolverhampton, Wolverhampton, UK

${ }^{3}$ Department of Practice and Policy, University College London, London, UK ${ }^{4}$ School of Health Administration, Anhui Medical University, China

${ }^{5}$ New Cross Hospital, The Royal Wolverhampton NHS Trust, UK

${ }^{6}$ Department of Public Health, Wolverhampton City Council, Wolverhampton, UK

${ }^{7}$ Department of Psychiatry, University of Liverpool, Liverpool, UK ${ }^{8}$ Dementia Collaborative Research Centre-Early Diagnosis and Prevention, Centre for Research on Ageing, Health and Wellbeing, Research School of Population Health, The Australian National University, Canberra, Australia

Acknowledgements RC and LW thank Alzheimer's Research and BUPA Foundation UK to provide research grants for the Research Programme of Dementia in China, particularly focusing on the early detection of dementia in older people. KJA is funded by NHMRC Fellowship \#1002560 and acknowledges support from the Dementia Collaborative Research Centres.

Contributors $\mathrm{RC}$ created a research idea for this study and made its conception and design. $\mathrm{LL}$ and $\mathrm{AC}$ were involved in the conception and design of this study, and contributed to the literature search and review. ID and WZ searched and reviewed the literature of 2013-2016. LW and DZ and $\mathrm{RC}$ were responsible for quantitative data analysis. JC, KJA, DL and GA contributed, along with all other authors, equally to the interpretation of the results and discussion of their implications. All authors were substantially involved in the writing and revisions of this manuscript, and all have given approval of the final version.

\section{Competing interests None declared.}

Provenance and peer review Not commissioned; externally peer reviewed.

Data sharing statement No additional data are available.

Open Access This is an Open Access article distributed in accordance with the Creative Commons Attribution Non Commercial (CC BY-NC 4.0) license, which permits others to distribute, remix, adapt, build upon this work noncommercially, and license their derivative works on different terms, provided the original work is properly cited and the use is non-commercial. See: http:// creativecommons.org/licenses/by-nc/4.0/

\section{REFERENCES}

1. Sternberg SA, Wolfson C, Baumgarten M. Undetected dementia in community-dwelling older people: the Canadian Study of Health and Aging. J Am Geriatr Soc 2000;48:1430-4.

2. Prince M, Bryce R, Ferri C. World Alzheimer Report 2011: the benefits of early diagnosis and intervention. Alzheimer's Disease International, 2011.

3. Bishop NA, Lu T, Yankner BA. Neural mechanisms of ageing and cognitive decline. Nature 2010;464:529-35.

4. Iliffe S, Robinson L, Brayne C, et al. Primary care and dementia: 1. diagnosis, screening and disclosure. Int $J$ Geriatr Psychiatry 2009;24:895-901.

5. Connolly A, Gaehl E, Martin H, et al. Underdiagnosis of dementia in primary care: variations in the observed prevalence and comparisons to the expected prevalence. Aging Ment Health 2011;15:978-84.

6. Chen R, Hu Z, Chen RL, et al. Determinants for undetected dementia and late-life depression. Br J Psychiatry 2013;203:203-8.

7. Savva GM, Arthur A. Who has undiagnosed dementia? A cross-sectional analysis of participants of the Aging, Demographics and Memory Study. Age Ageing 2015;44:642-7.

8. Ross GW, Abbott RD, Petrovitch $\mathrm{H}$, et al. Frequency and characteristics of silent dementia among elderly JapaneseAmerican men: the Honolulu-Asia Aging Study. JAMA 1997;277:800-5.

9. Rahkonen T, Luukkainen-Markkula R, Paanila S, et al. Delirium episode as a sign of undetected dementia among community 
dwelling elderly subjects: a 2 year follow up study. J Neurol Neurosurg Psychiatry 2000;69:519-21.

10. Bekkelund SI, Kujala I, Rosenvinge B. Unrecognized dementia in elderly patients admitted to hospital with psychiatric symptoms. $J$ Geriatr Psychiatry Neurol 2001;14:7-10.

11. Verhey FR, Jolles J, Ponds RW, et al. Diagnosing dementia: a comparison between a monodisciplinary and a multidisciplinary approach. J Neuropsychiatry Clin Neurosci 1993;5:78-85.

12. Lopponen MK, Isoaho RE, Raiha IJ, et al. Undiagnosed diseases in patients with dementia - a potential target group for intervention. Dement Geriatr Cogn Disord 2004;18:321-9.

13. Lopponen M, Raiha I, Isoaho R, et al. Diagnosing cognitive impairment and dementia in primary health care-a more active approach is needed. Age Ageing 2003;32:606-12.

14. Wells GA, O'Connell D, Peterson J, et al. The Newcastle-Ottawa Scale (NOS) for assessing the quality of nonrandomised studies in meta-analyses. 2014; http://www.ohri.ca/programs/clinical_ epidemiology/oxford.asp (accessed 09 Feb 2016).

15. Chen R, Copeland JR, Wei L. A meta-analysis of epidemiological studies in depression of older people in the People's Republic of China. Int J Geriatr Psychiatry 1999;14:821-30.

16. O'Connor DW, Pollitt PA, Hyde JB, et al. Do general practitioners miss dementia in elderly patients? BMJ 1988;297:1107-10.

17. Lagaay AM, van der Meij JC, Hijmans W. Validation of medical history taking as part of a population based survey in subjects aged 85 and over. BMJ 1992;304:1091-2.

18. Worrall G, Moulton N. Cognitive function. Survey of elderly persons living at home in rural Newfoundland. Can Fam Physician 1993;39:772-7.

19. Callahan CM, Hendrie HC, Tierney WM. Documentation and evaluation of cognitive impairment in elderly primary care patients. Ann Intern Med 1995;122:422-9.

20. Eefsting JA, Boersma F, Van den Brink W, et al. Differences in prevalence of dementia based on community survey and general practitioner recognition. Psychol Med 1996;26:1223-30.

21. Valcour VG, Masaki KH, Curb JD, et al. The detection of dementia in the primary care setting. Arch Intern Med 2000;160:2964-8.

22. Zunzunegui Pastor MV, Del Ser T, Rodríguez Laso A, et al. Demencia no detectada y utilización de los servicios sanitarios: implicaciones para la atención primaria. Aten Primaria 2003;31:581-6.

23. Boise L, Neal MB, Kaye J. Dementia assessment in primary care: results from a study in three managed care systems. J Gerontol A Biol Sci Med Sci 2004;59:M621-6.

24. Boustani M, Callahan CM, Unverzagt FW, et al. Implementing a screening and diagnosis program for dementia in primary care. $J$ Gen Intern Med 2005;20:572-7.

25. Borson S, Scanlan JM, Watanabe J, et al. Improving identification of cognitive impairment in primary care. Int $J$ Geriatr Psychiatry 2006;21:349-55.

26. Chan DC, Kasper JD, Black BS, et al. Clinical diagnosis of dementia, not behavioral and psychologic symptoms, is associated with psychotropic drug use in community-dwelling elders classified as having dementia. J Geriatr Psychiatry Neurol 2007;20:100-6.
27. Wilkins $\mathrm{CH}$, Wilkins $\mathrm{KL}$, Meisel $\mathrm{M}$, et al. Dementia undiagnosed in poor older adults with functional impairment. J Am Geriatr Soc 2007;55:1771-6.

28. Jitapunkul S, Chansirikanjana S, Thamarpirat J. Undiagnosed dementia and value of serial cognitive impairment screening in developing countries: a population-based study. Geriatr Gerontol Int 2009;9:47-53.

29. Eichler T, Thyrian JR, Hertel J, et al. Rates of formal diagnosis of dementia in primary care: The effect of screening. Alzheimers Dement (Amst) 2015;1:87-93.

30. Olafsdottir M, Skoog I, Marcusson J. Detection of dementia in primary care: the Linköping study. Dement Geriatr Cogn Disord 2000;11:223-9.

31. Nygaard HA, Ruths S. Missing the diagnosis: senile dementia in patients admitted to nursing homes. Scand J Prim Health Care 2003;21:148-52.

32. Magsi H, Malloy $\mathrm{T}$. Underrecognition of cognitive impairment in assisted living facilities. J Am Geriatr Soc 2005;53: 295-8.

33. Collerton J, Davies K, Jagger C, et al. Health and disease in 85 year olds: baseline findings from the Newcastle 85 cohort study. BMJ 2009;339:b4904.

34. Ferretti M, Seematter-Bagnoud L, Martin E, et al. New diagnoses of dementia among older patients admitted to postacute care. J Am Med Dir Assoc 2010;11:371-6.

35. Lithgow S, Jackson GA, Browne D. Estimating the prevalence of dementia: cognitive screening in Glasgow nursing homes. Int J Geriatr Psychiatry 2012;27:785-91.

36. Bartfay E, Bartfay WJ, Gorey KM. Prevalence and correlates of potentially undetected dementia among residents of institutional care facilities in Ontario, Canada, 2009-2011. Int J Geriatr Psychiatry 2013;28:1086-94.

37. World Bank. Country and Lending Groups. http://data.worldbank.org/ about/country-and-lending-groups

38. Prince MJ. The 10/66 dementia research group-10 years on. Indian J Psychiatry 2009;51(Suppl 1):S8-S15.

39. Putting dementia on the map and driving up standards of care. https://www.gov.uk/government/news/putting-dementia-on-the-mapand-driving-up-standards-of-care

40. Shah A, Lindesay J, Jagger C. Is the diagnosis of dementia stable over time among elderly immigrant Gujaratis in the United Kingdom (Leicester)? Int J Geriatr Psychiatry 1998;13:440-4.

41. Livingston G, Leavey G, Kitchen G, et al. Accessibility of health and social services to immigrant elders: the Islington Study. Br J Psychiatry 2002;180:369-73.

42. van den Dungen $P$, van Marwijk HWM, van der Horst HE, et al. The accuracy of family physicians' dementia diagnoses at different stages of dementia: a systematic review. Int J Geriatr Psychiatry 2012;27:342-54.

43. Chen $\mathrm{R}, \mathrm{Ma} \mathrm{Y}$, Wilson $\mathrm{K}$, et al. A multicentre community-based study of dementia cases and subcases in older people in rural and urban China-the GMS-AGECAT prevalence and socio-economic correlates. Int J Geriatr Psychiatry 2012;27:692-702. 